Electrochemistry Communications

Elsevier Editorial system(tm) for

Manuscript Draft

Manuscript Number: EC15-1371R2

Title: Electrochemical preparation and characterization of magnetic coreshell nanowires for biomedical applications

Article Type: Short Communication

Keywords: electrochemical synthesis; coreeshell magnetic nanorods; functionalization; cellular viability

Corresponding Author: Prof. Elisa Vallés, PhD

Corresponding Author's Institution: Universitat de Barcelona

First Author: Clàudia Gispert, Master

Order of Authors: Clàudia Gispert, Master; Albert Serrà, PhD student; Maria Elisa Alea, PhD student; Mafalda Rodrigues, Dr.; Elvira Gómez, Dr.; Margarita Mora, Dr.; Maria Lluïsa Sagristà, Dr.; Maria Luisa Pérez, Dr.; Elisa Vallés, PhD

Abstract: Magnetic CoNi@Au core-shell nanorods have been electrochemically synthesized, characterized and functionalized to test their inherent citotoxycity in order to assess their potential use for biomedical applications. The initially electrodeposited CoNi nanorods have been covered with a gold layer by means of galvanic displacement to minimize the nanowires toxicity and their aggregation, and favour the functionalization. The presence of a gold layer on the nanorod surface slightly modifies the magnetic behaviour of the as-deposited nanorods, maintaining their soft-magnetic behaviour and high magnetization of saturation. The complete covering of the nanorods with the gold shell favours a good functionalization with a layer of (11-

Mercaptoundecyl) hexa (ethylene glycol) molecules, in order to create a hydrophilic coating to avoid the aggregation of nanorods, keeping them in suspension and give them stability in biological media. The presence of the organic layer incorporated was detected by means of electrochemical probe experiments. A cytotoxicity test of functionalized core-shell nanorods, carried out with adherent Hela cells, showed that cell viability was higher than $80 \%$ for amounts of nanorods up to $10 \mu \mathrm{g} \mathrm{mL}-1$. These results make functionalized nanorods promising vehicles for targeted drug delivery in medicine, which gives a complementary property to the magnetic nanoparticles. 
Ms. Ref. No.: EC15-1371R1

Title: Electrochemical preparation and characterization of magnetic core-shell nanowires for biomedical applications

Electrochemistry Communications

Dear Prof. Comptom

I'm pleased to send you the Revised version 2 of the manuscript Ref. No.: EC15-1371R1. It seems that Reviewer 2 is still critic with two questions of the manuscript. In the first revision, we tried to clearly give answers to the questions, but probably, our justifications were not clear enough. Therefore, according to Referee 2 :

-We have now introduced a sentence in the text justifying the expected low toxicity of our materials.

-We answer and justify the use of the electrochemical test for the detection of a complete layer of gold

-We send and interpret the EDS spectra of the NRs

We hope that now the manuscript could be accepted for publication in Electrochem. Commun. W

Best regards

Elisa Vallés

Reviewer's comments

\section{$\underline{\text { Reviewer } 2}$}

The revised version did not adequately address the concerns I raised in my previous report. I agree with the statement that Co release from implants might be more problematic than from cancer treatment drug delivery robots - but a sentence addressing this general concern should be included in the text.

Accordingly to the reviewer's comment we have included a sentence addressed to the utility of using our proposed nanostructures for cancer therapy. We include this sentence after the results of cell viability of our nanostructures. The sentence introduced was: "Co-based materials are not usually selected for implants in biomedical applications, due to their potential toxicity; however, their protection with gold and organic molecules makes them potentially useful as drug delivery robots for therapies as cancer treatment. Moreover, the amount of metal delivered to an organism when it is used as drug carrier is very low and the carrier is usually eliminated from the body in a relatively short time. Therefore, CoNi@Au 
nanostructures could be proposed as promising magnetic nano-carriers for cancer treatments."

My major concern is still that there is no conclusive proof that the particles have been fully covered with $\mathrm{Au}$. The authors claim that $\mathrm{Co}$ and Ni dissolve in the $\mathrm{H} 2 \mathrm{SO} 4$ spontaneously before the $\mathrm{CV}$ is started when there is no Au shell. However, the Au peak that is recorded on the coreshell particles does not prove that there are any of the cores left. Assuming there are holes in the u shell the CoNi core would dissolve out of the core-shell-particle and the CV would only show the response of the empty Au shell. There is therefore no proof provided that the Au shell is indeed fully covering and protecting the CoNi core in my opinion.

Really, we propose the electrochemical test in $\mathrm{H}_{2} \mathrm{SO}_{4}$ to determine the full covering of our nanorods with a thin gold layer. When the nanorods are placed on the surface of the vitreous carbon electrode and immersed in the $\mathrm{H}_{2} \mathrm{SO}_{4}$ solution, we observe two different behaviors, depending on the fully coverage of the NRs with the gold layer:

- When the gold layer does not exist or it is not complete (short times of galvanic displacement) the CoNi nanorods dissolve in the medium, disappear of the vitreous carbon surface and the voltammetric response of the nanorods is null. Only the response of a bare vitreous carbon electrode (no faradaic current) in the sulfuric solution is observed.

- When the gold layer fully covers the nanorods, the gold response is observed. This response maintains for several successive cycles in the sulfuric medium, without loss of signal. Repetitive experiments with the same amount of nanowires on the vitreous carbon substrate show the same voltammetric response, which correspond to the same amount of gold covering the nanorods. If the gold layer has holes (not complete layer), the CoNi core actually dissolves and the thin layer of gold (nanometric) does not maintain without the core. The fragments of gold must fall of the surface of the glassy carbon during successive scans and golden profile is not observed

In the interesting paper of Compton et al (Advanced Functional Materials, 2015) that the referee comments, the test of covering of NPs with a gold layer allows to determine the voltammetric response of the core (iron oxides) or the shell (gold). In our different electrochemical test, the aggressive medium used $\left(\mathrm{H}_{2} \mathrm{SO}_{4} 0.5 \mathrm{M}\right)$ leads to the total dissolution of the CoNi core when gold doesn't fully cover the core.

I also still do not understand why the authors refuse to present the EDS spectra they refer to in the text. From these it could be clearly shown that the $O$ content of the samples is small. Stating that some other author who used the same core and shell materials but different shapes and reaction conditions obtained dense shells is not sufficient to address this concern.

For the above reasons I still do not consider the manuscript to be suitable for publication in Electrochemistry Communications in the present state. 
We had not introduced the EDS spectra in the manuscript due to space limitations, i.e. the restriction of the words and figures inherent to the letter type of the proposed article. However, we have not any problem in send them to the reviewer (included here).
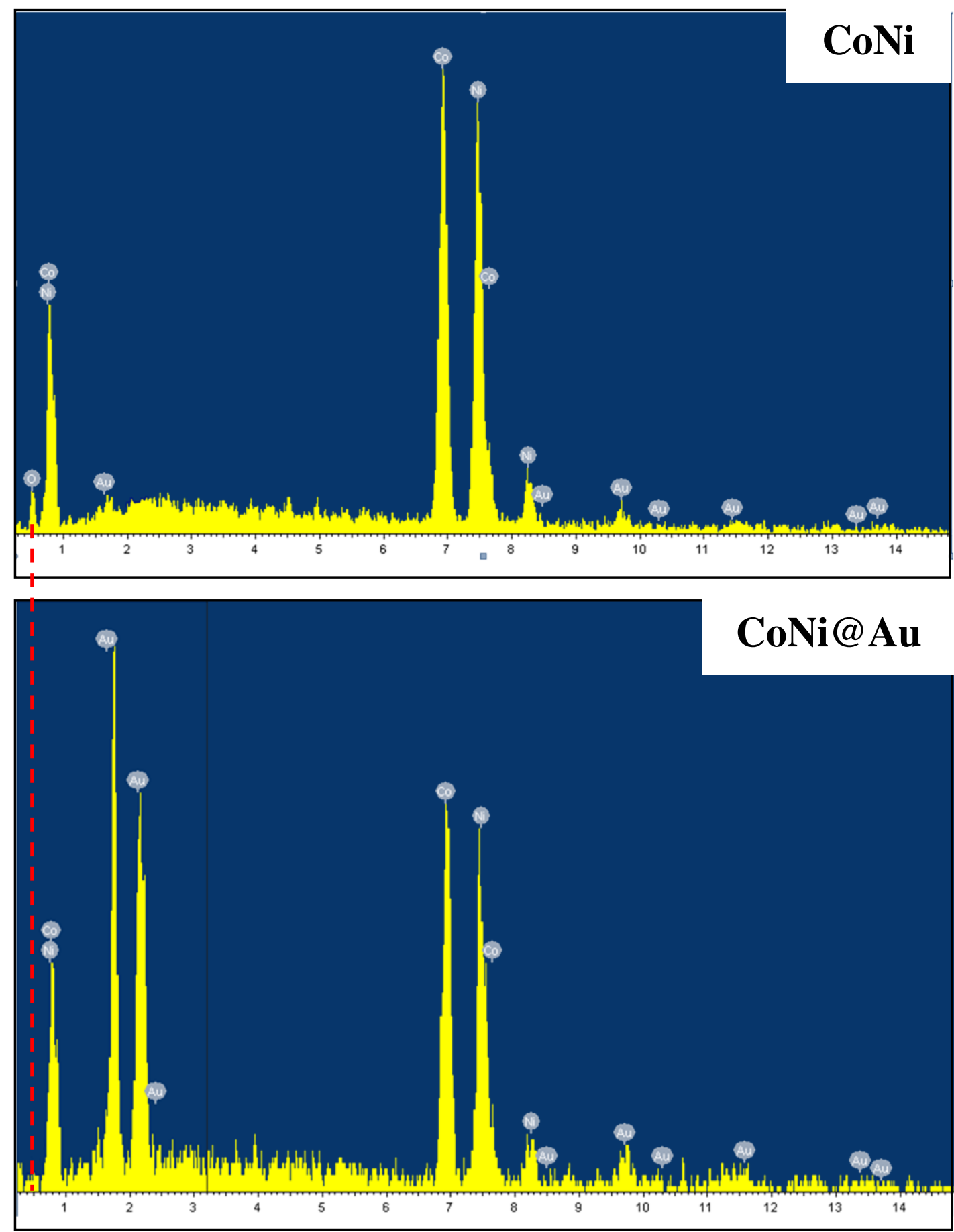

The EDS spectrum of the CoNi NRs shows the peaks of the Co and Ni elements, being small the signal corresponding to O. When they covered with the gold layer (CoNi@Au), the signals assigned to $\mathrm{Co}, \mathrm{Ni}$ and $\mathrm{Au}$ were detected. The low amount of $\mathrm{O}$ detected allows justifying the galvanic displacement of the metals by gold atoms. 
Moreover, it has been demonstrated that although some superficial oxides are formed on cobalt or nickel nanostructures, the complete formation of gold or platinum layers over these structures is possible using the galvanic displacement method, because the atoms of precious metals favor the removal of oxygen from the cobalt surface and, then, the galvanic displacement [Surf. Sci 606 (2012) 634]. S. Grau et al. Handbook of Nanoelectrochemistry, 2015, Springer International Publishing, DOI: 10.1007/978-3-319-15207-3-36-1]. Moreover, when Co@Pt or Ni@Pt structures are prepared for electrocatalysis and the resulting core-shell particles (superficially oxidized) are activated in $\mathrm{H}_{2} \mathrm{SO}_{4}$, total recovery of the particles by the precious metal shell has been proved by several authors.

The use of a thicker layer of gold to protect the nanowires will imply using different methods to obtain the shell layer, as electrodeposition, which supposes a higher complexity that the use of galvanic displacement to obtain the shell that, although thinner, is sufficient for protection and functionalization of the NRs.

We hope that now the referee can consider that we have justified more clearly our proposals. 
Reviewer's comments

\section{$\underline{\text { Reviewer } 2}$}

The revised version did not adequately address the concerns I raised in my previous report.

In the first revision, we tried to clearly give answers to the questions, but probably, our justifications were not clear enough. We hope that now the referee can consider that we have justified more clearly our proposals, and that the paper has been improved by the incorporation of a new sentence:

I agree with the statement that Co release from implants might be more problematic than from cancer treatment drug delivery robots - but a sentence addressing this general concern should be included in the text.

Accordingly to the reviewer's comment we have included a sentence addressed to the utility of using our proposed nanostructures for cancer therapy. We include this sentence after the results of cell viability of our nanostructures. The sentence introduced was: "Co-based materials are not usually selected for implants in biomedical applications, due to their potential toxicity; however, their protection with gold and organic molecules makes them potentially useful as drug delivery robots for therapies as cancer treatment. Moreover, the amount of metal delivered to an organism when it is used as drug carrier is very low and the carrier is usually eliminated from the body in a relatively short time. Therefore, CoNi@Au nanostructures could be proposed as promising magnetic nano-carriers for cancer treatments."

My major concern is still that there is no conclusive proof that the particles have been fully covered with $\mathrm{Au}$. The authors claim that $\mathrm{Co}$ and $\mathrm{Ni}$ dissolve in the $\mathrm{H} 2 \mathrm{SO} 4$ spontaneously before the $\mathrm{CV}$ is started when there is no Au shell. However, the Au peak that is recorded on the coreshell particles does not prove that there are any of the cores left. Assuming there are holes in the u shell the CoNi core would dissolve out of the core-shell-particle and the CV would only show the response of the empty Au shell. There is therefore no proof provided that the Au shell is indeed fully covering and protecting the CoNi core in my opinion.

Really, we propose the electrochemical test in $\mathrm{H}_{2} \mathrm{SO}_{4}$ to determine the full covering of our nanorods with a thin gold layer. When the nanorods are placed on the surface of the vitreous carbon electrode and immersed in the $\mathrm{H}_{2} \mathrm{SO}_{4}$ solution, we observe two different behaviors, depending on the fully coverage of the NRs with the gold layer:

- When the gold layer does not exist or it is not complete (short times of galvanic displacement) the CoNi nanorods dissolve in the medium, disappear of the vitreous carbon surface and the voltammetric response of the nanorods is null. Only the response of a bare vitreous carbon electrode (no faradaic current) in the sulfuric solution is observed. 
- When the gold layer fully covers the nanorods, the gold response is observed. This response maintains for several successive cycles in the sulfuric medium, without loss of signal. Repetitive experiments with the same amount of nanowires on the vitreous carbon substrate show the same voltammetric response, which correspond to the same amount of gold covering the nanorods. If the gold layer has holes (not complete layer), the CoNi core actually dissolves and the thin layer of gold (nanometric) does not maintain without the core. The fragments of gold must fall of the surface of the glassy carbon during successive scans and golden profile is not observed

In the interesting paper of Compton et al (Advanced Functional Materials, 2015) that the referee comments, the test of covering of NPs with a gold layer allows to determine the voltammetric response of the core (iron oxides) or the shell (gold). In our different electrochemical test, the aggressive medium used $\left(\mathrm{H}_{2} \mathrm{SO}_{4} 0.5 \mathrm{M}\right)$ leads to the total dissolution of the CoNi core when gold doesn't fully cover the core.

I also still do not understand why the authors refuse to present the EDS spectra they refer to in the text. From these it could be clearly shown that the $O$ content of the samples is small. Stating that some other author who used the same core and shell materials but different shapes and reaction conditions obtained dense shells is not sufficient to address this concern.

For the above reasons I still do not consider the manuscript to be suitable for publication in Electrochemistry Communications in the present state.

We had not introduced the EDS spectra in the manuscript due to space limitations, i.e. the restriction of the words and figures inherent to the letter type of the proposed article. However, we have not any problem in send them to the reviewer (included here). 

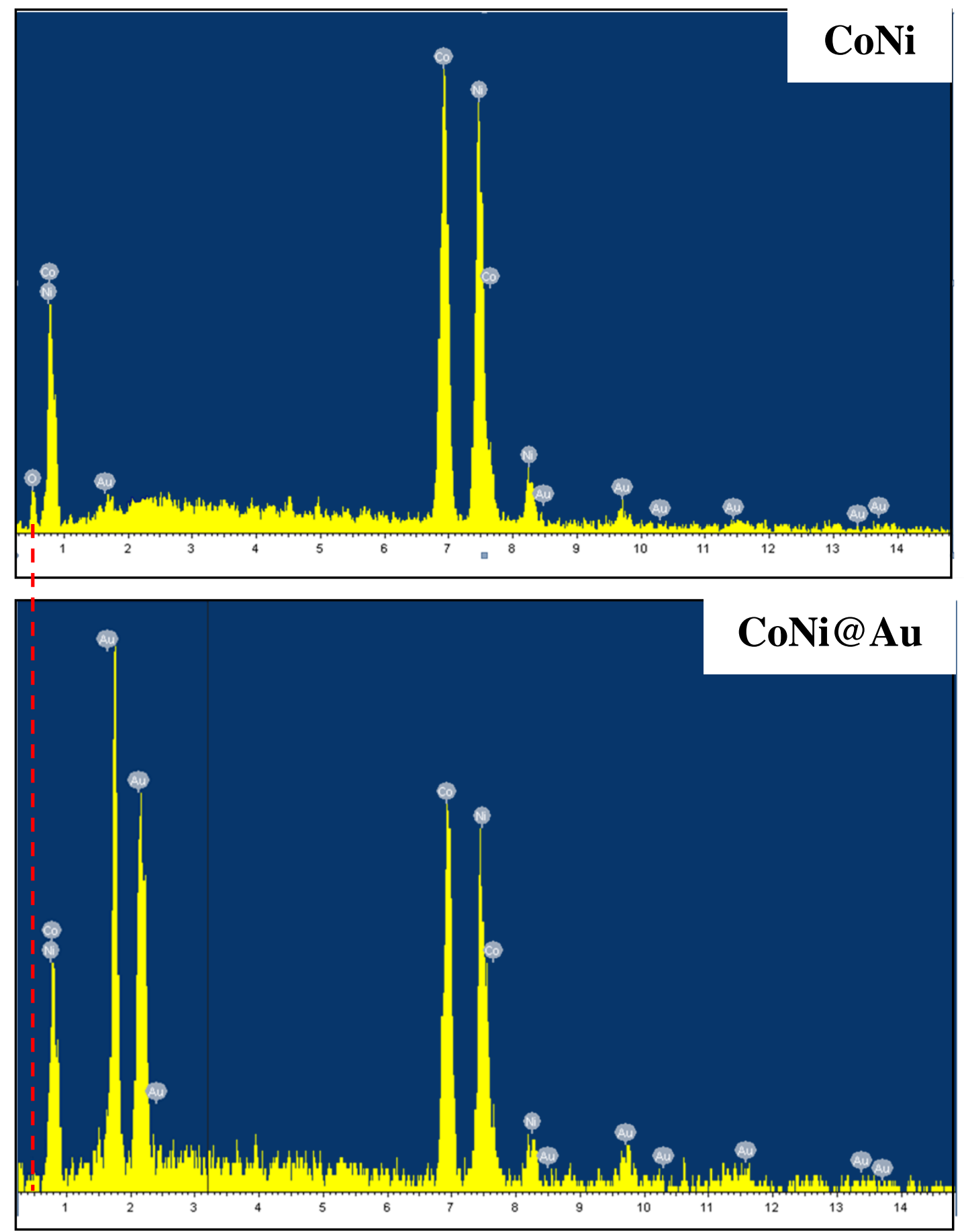

The EDS spectrum of the CoNi NRs shows the peaks of the Co and Ni elements, being small the signal corresponding to $\mathrm{O}$. When they covered with the gold layer (CoNi@Au), the signals assigned to $\mathrm{Co}, \mathrm{Ni}$ and $\mathrm{Au}$ were detected. The low amount of $\mathrm{O}$ detected allows justifying the galvanic displacement of the metals by gold atoms.

Moreover, it has been demonstrated that although some superficial oxides are formed on cobalt or nickel nanostructures, the complete formation of gold or platinum layers over these structures is possible using the galvanic displacement method, because the atoms of precious metals favor the removal of oxygen from the cobalt surface and, then, the galvanic 
displacement [Surf. Sci 606 (2012) 634]. S. Grau et al. Handbook of Nanoelectrochemistry, 2015, Springer International Publishing, DOI: 10.1007/978-3-319-15207-3-36-1]. Moreover, when Co@Pt or Ni@Pt structures are prepared for electrocatalysis and the resulting core-shell particles (superficially oxidized) are activated in $\mathrm{H}_{2} \mathrm{SO}_{4}$, total recovery of the particles by the precious metal shell has been proved by several authors.

The use of a thicker layer of gold to protect the nanowires will imply using different methods to obtain the shell layer, as electrodeposition, which supposes a higher complexity that the use of galvanic displacement to obtain the shell that, although thinner, is sufficient for protection and functionalization of the NRs. 
Dear Editor of Electrochem. Commun.

Regarding the reason for urgent publication of the manuscript:

We demonstrate in the work that core@shell magnetic nanorods (few microns long and around $120 \mathrm{~nm}$ of diameter), electrochemically prepared, can be useful for possible biomedical application, because they can be effectively funcionalized and the cellular viability in their presence is high. It's the first time that CoNi@Au nanorods are proposed as potential magnetic transporters of functional molecules.

Electrochemical methods have been used to synthesize the magnetic nanorods, to cover them with a gold thin layer and to detect the needed conditions to obtain a complete layer of gold that allows the functionalization of the rods and their protection in the presence of an aggressive medium. Also, an electrochemical probe is proposed to corroborate the nanorods functionalization.

We send color figures for on line publication and the black and white ones for standard publication.

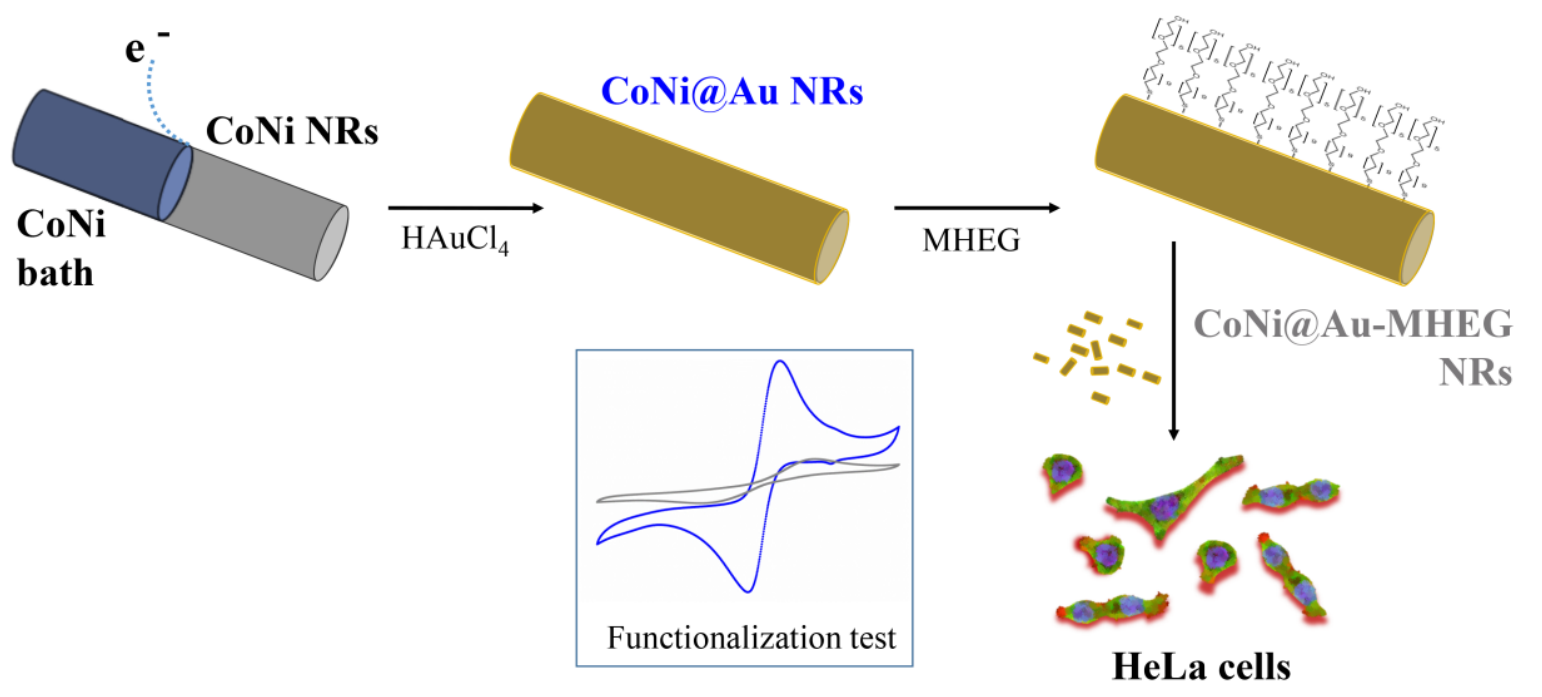

Looking forward to hearing from you.

Yours sincerely,

Dra. Elisa Vallés 

Juan M. Feliu

Dpto. Química Física, Universidad de Alicante

juan.feliu@ua.es

Expert electrochemist and controlled nanostructures

Editor of J. Electroanal. Chem.

Ivani A. Carlos

Departamento de Química, Universidade Federal de Sao Carlos, CP 676, 13585-905, Sao Carlos-SP, Brazil

e-mail: diac@ufscar.br 


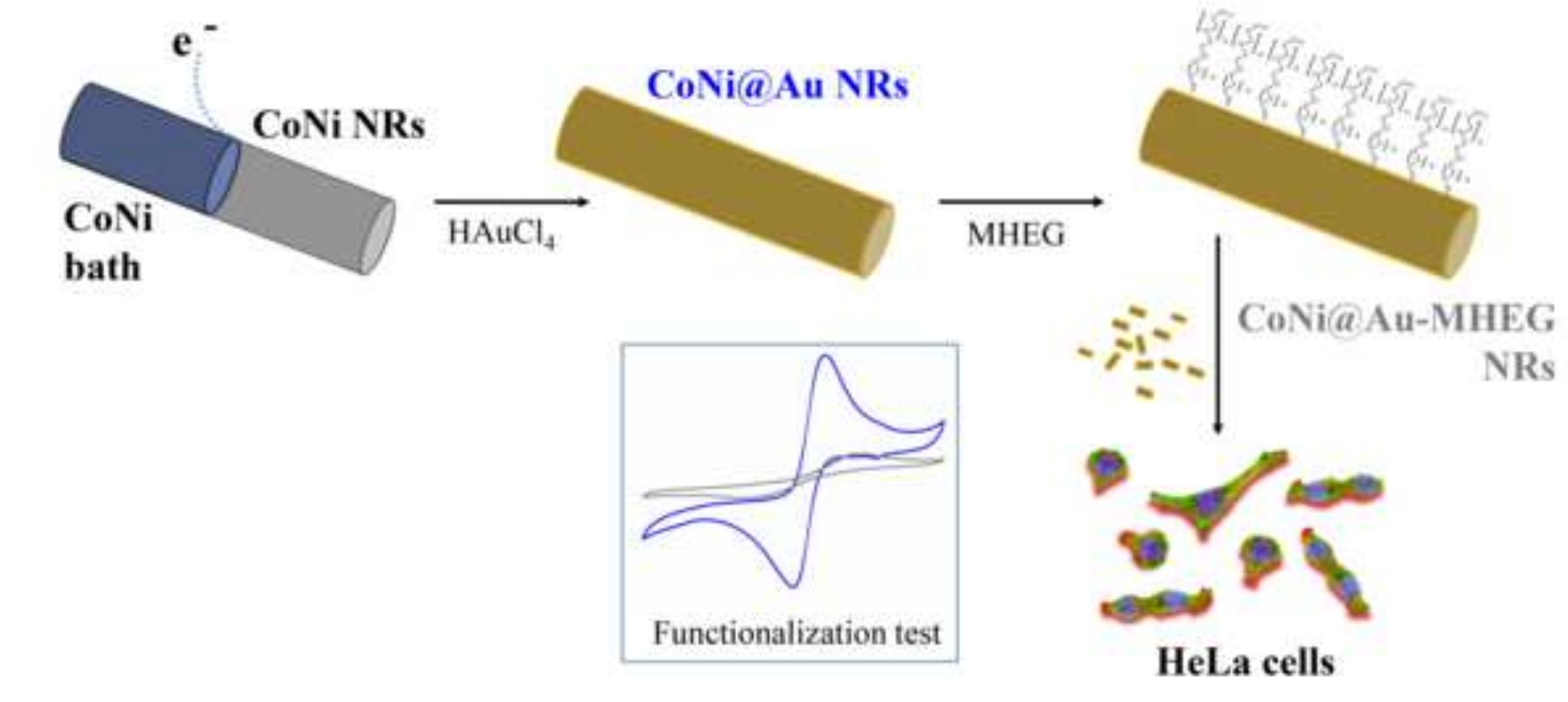

HeLa cells 


\section{Highlights}

- Electrochemical procedure for magnetic CoNi@Au nanorods preparation

- Electrochemical test of complete Au layer formation

- Good functionalization of the nanorods for stability in solution and biocompatibility

- Proposal of electrochemical test of the functionalization

- New promising magnetic vehicles for biomedical application 


\title{
Electrochemical preparation and characterization of magnetic core-shell nanowires for biomedical applications
}

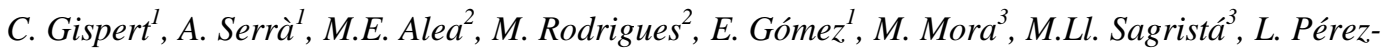 \\ García $^{2,4}$, E. Vallés $^{1 *}$ \\ ${ }^{l}$ Ge-CPN, Dept. Química Física, Institut de Nanociència i Nanotecnologia $\left(I N^{2} U B\right)$, Universitat de \\ Barcelona (UB), Martí i Franquès 1, 08028 Barcelona. \\ ${ }^{2}$ Dept. Farmacologia i Química Terapèutica, IN ${ }^{2} U B, U B, A v d a$. Joan XXIII s/n, 08028 Barcelona. \\ ${ }^{3}$ Dept. Bioquímica i Biologia Molecular (Facultat de Biologia), UB, Avda. Diagonal 643, 08028 \\ Barcelona \\ ${ }^{4}$ Present address: School of Pharmacy, The University of Nottingham, University Park, Nottingham NG7 \\ $2 R D$, United Kingdom
}

*Corresponding author: E. Vallés, e-mail: $\underline{\text { evalles@ub.edu }}$

\begin{abstract}
Magnetic CoNi@Au core-shell nanorods have been electrochemically synthesized, characterized and functionalized to test their inherent citotoxycity in order to assess their potential use for biomedical applications. The initially electrodeposited CoNi nanorods have been covered with a gold layer by means of galvanic displacement to minimize the nanowires toxicity and their aggregation, and favour the functionalization. The presence of a gold layer on the nanorod surface slightly modifies the magnetic behaviour of the as-deposited nanorods, maintaining their soft-magnetic behaviour and high magnetization of saturation. The complete covering of the nanorods with the gold shell favours a good functionalization with a layer of (11-Mercaptoundecyl)hexa(ethylene glycol) molecules, in order to create a hydrophilic coating to avoid the aggregation of nanorods, keeping them in suspension and give them stability in biological media. The presence of the organic layer incorporated was detected by means of electrochemical probe experiments. A cytotoxicity test of functionalized core-shell nanorods, carried out with adherent HeLa cells, showed that cell viability was higher than $80 \%$ for amounts of nanorods up to $10 \mu \mathrm{g} \mathrm{mL}^{-1}$. These results make functionalized nanorods promising vehicles for targeted drug delivery in medicine, which gives a complementary property to the magnetic nanoparticles.
\end{abstract}

\section{Keywords}

Electrochemical synthesis, core@shell magnetic nanorods, functionalization, cellular viability

\section{Introduction}

The electrochemical methods have been recently demonstrated as very useful for the synthesis of metallic nanostructures as nanoparticles, nanotubes or nanorods [1-2], which have been applied in catalysis [3], sensing [4] or computer science [5]. On the other hand, magnetic nanoparticles, especially of iron oxides, are being used in biomedicine for treatment as hyperthermia or drug delivery [6-9]. Recently, the possibility of using nickel nanowires in biomedicine has been also proposed [10-12]. In the present work we propose the test of CoNi@Au nanorods (NRs), of a few microns of length, as magnetic supports that could be functionalized and show little cellular toxicity.

CoNi NRs present high magnetization of saturation and chemical stability, being good magnetic vehicles for the transport of therapeutic molecules. Moreover, the shape of these nanostructures can present the advantage, respect to nanoparticles, of improving the therapeutic molecules/vehicle ratio. However, 
structures of less than $5 \mu \mathrm{m}$ and nanometric diameter will be prepared, in order to permit their circulation by the blood vessels in possible medical applications. In order to favour both the biocompatibility and the functionability of the nanorods, a complete thin layer of gold is induced on the surface, being its correct formation electrochemically tested. The core@shell NRs are functionalized with (11Mercaptoundecyl)hexa(ethylene glycol) (MHEG) to make hydrophilic their surface, minimize the aggregation of the magnetic nanorods and favour the interaction with the cells present in the culture. The viability of HeLa cells in the presence of the CoNi@Au-MHEG NRs will be analysed.

\section{Experimental}

Nanorods preparation and electrochemical tests were carried out at room temperature using a potentiostat/galvanostat Autolab PGSTAT30 (GPES software), and a cell with three electrodes, being a $\mathrm{Ag} / \mathrm{AgCl} / \mathrm{KCl} 3 \mathrm{M}$ the reference and a platinum spiral the auxiliary electrode.

The nanorods were prepared in Millipore polycarbonate membranes $(20 \mu \mathrm{m}$-thick, $100 \mathrm{~nm}$ nominal pore size diameter), metalized by vacuum evaporation (in IMB-CNM.CSIC of Barcelona) with gold (100 nm-thick) on one side, using a $\mathrm{CoCl}_{2} 0.2 \mathrm{M}+\mathrm{NiCl}_{2} 0.9 \mathrm{M}+\mathrm{H}_{3} \mathrm{BO}_{3} 0.5 \mathrm{M}, \mathrm{pH}=3$ solution. The synthesized nanorods were exhaustively cleaned in chloroform. The gold layer formation was carried out by means galvanic displacement, by immersing the nanorods in a $\mathrm{HAuCl}_{4} 2.9 \mathrm{mM}$ solution during different times. The complete formation of the gold shell was tested by placing some nanorods in a vitreous carbon electrode $(2 \mathrm{~mm}$ diameter $)$ and testing their voltammetric response in $\mathrm{a}_{2} \mathrm{SO}_{4} 0.5 \mathrm{M}$ solution. In all cases, Argon flow was used before each experiment, maintaining argon atmosphere during the study. The solutions were always prepared with Millipore MilliQ water $\left(18.2 \mathrm{M} \Omega \cdot \mathrm{cm}^{-1}\right)$.

The nanorods were examined by means of a Field Emission Scanning Electron Microscopy FE-SEM (JSM-7100F). An X-ray analyzer (EDS) incorporated in a Leica Stereo Scan S-360 Equipment was used to determine the elemental composition. The magnetic behaviour was analysed by means a SQUID magnetometer at $300 \mathrm{~K}$.

To improve the solubility and biocompatibility of the nanorods, $150 \mu \mathrm{g}$ of nanorods were functionalized with $1 \mathrm{~mL}$ of $8 \mathrm{mM}$ MHEG solution in ethanol overnight, with shaking at room temperature. The nanorods were then washed with equal volume of ethanol 3 times with sonication for 5 seconds. The presence of MHEG molecules on the CoNi@Au NRs has been corroborated by means of electrochemical probe experiments, by recording cyclic voltammetries of the $\mathrm{Fe}(\mathrm{II}) / \mathrm{Fe}(\mathrm{III})$ system in a $\mathrm{KNO}_{3} 0.2 \mathrm{M}+2$ $\mathrm{mM} \mathrm{K}_{4}\left[\mathrm{Fe}(\mathrm{CN})_{6}\right]+2 \mathrm{mM} \mathrm{K}_{3}\left[\mathrm{Fe}(\mathrm{CN})_{6}\right]$ solution in CoNi@Au and in CoNi@Au-MHEG NRs. For each experiment, $2.5 \mu \mathrm{L}$ of a NRs suspension $(15 \mu \mathrm{g}$ in $1 \mathrm{~mL}$ of a water-ethanol mixture) were dropped on the surface of a glassy carbon $(\mathrm{GC}) \operatorname{rod}\left(0.031 \mathrm{~cm}^{2}\right.$ of diameter) and dried under nitrogen.

Human HeLa cervical adenocarcinoma cells (ATCC CCL-2) were used to test the cytotoxicity of nanorods. HeLa cells were cultured at $37{ }^{\circ} \mathrm{C}$ in a humidified sterile atmosphere of $95 \%$ air and $5 \% \mathrm{CO}_{2}$, using complete growth medium (DMEM) supplemented with fetal bovine serum $(10 \% \mathrm{v} / \mathrm{v})$, glucose $(4.5$ $\left.\mathrm{g} \mathrm{L}^{-1}\right)$, L-glutamine $\left(292 \mathrm{mg} \mathrm{L}^{-1}\right)$, streptomycin sulfate $\left(50 \mathrm{mg} \mathrm{L}^{-1}\right)$ and potassium penicillin $\left(50000 \mathrm{U} \mathrm{L}^{-}\right.$ ${ }^{1}$ ). All the media, sera and antibiotics were provided by Gibco-Life Technologies (Paisley, UK). Cells were seeded in 96-well plates (3300 cells well ${ }^{-1}$, Luna Automated Cell Counter) and grown up to 70-85\% confluence. Cells were then incubated with different concentrations of CoNi@Au-MHEG nanorods (from 0.003 to $52 \mu \mathrm{g} \mathrm{mL}^{-1}$ ) for $24 \mathrm{~h}$. Cells incubated with the complete medium in the absence of nanorods were used as control. After incubation, the medium was discarded, cells were washed three times with cold PBS and incubation was followed for 24 additional hours with fresh culture medium. Cell viability was determined by means of the 3-(4,5-dimethylthiazol-2-yl)-2,5-diphenyltetrazoliumbromide (MTT, SigmaAldrich) assay. Briefly, the remaining HeLa cells were incubated with $0.05 \mathrm{mg} \mathrm{MTT} \mathrm{mL}^{-1}$ in complete DMEM for $3 \mathrm{~h}$. The medium was discarded, formazan crystals were solubilized with pure DMSO and formazan concentration was determined by absorption at $\lambda_{\text {ex }}$ of $526 \mathrm{~nm}$ (microplate reader SynergyMx ${ }^{\mathrm{TM}}$, 
BioTek Instruments, Inc.). Cell viability was determined by the ratio between the absorbance of treated cells and that of non-treated cells (control, $100 \%$ viability).

\section{Results and discussion}

The protocol for the nanorods preparation was the following: 1) determination of the mass of the membrane, 2) immersion of the membrane in water during 24 hours, 3) electrodeposition of the CoNi NRs in the described solution at $-1.0 \mathrm{~V}$ and deposition charge of $4.4 \mathrm{C}$ for a geometrical area of $3.1 \mathrm{~cm}^{2}$ and a porosity of the $13.8 \%, 4$ ) determination of the mass of the membrane containing the nanorods, 5) removing of the gold layer of the membrane with a saturated $\mathrm{KI}_{3}$ solution, 6) removing of the membrane and cleaning $(10 \mathrm{x})$ of the nanorods with chloroform. Good reproducibility was obtained in successive synthesis, showing the Figure 1a a representative chronoamperometric curve of the electrodeposition. The shape of the curve reveals the initial nucleation and growth of the CoNi on the gold in the bottom of the membrane's channels, the posterior decay due to the depletion of the electroactive species near of the growing nanorods and a final stabilization of the current when a stationary regime of transport of the electroactive species in the interior of the channels is attained.

The SEM pictures of the as-deposited nanorods show their dimensions and good definition. CoNi NRs of 65 wt. $\%$ of Co, $110 \pm 15 \mathrm{~nm}$ of real diameter, $3.7 \pm 0.4 \mu \mathrm{m}$ and around 34 of aspect ratio were obtained (Figure 1a). These nanorods were immersed in the $\mathrm{Au}(\mathrm{III})$ solution for different times in order to obtain a complete gold layer on their surface. The necessary immersion time to cover all the rods was determined by means cyclic voltammetry of the CoNi@Au NRs in the $\mathrm{H}_{2} \mathrm{SO}_{4}$ solution: A drop of a NRs suspension in water-ethanol mixture was placed, in each case, on the top of a GC electrode and dried slowly with a nitrogen current flow. The GC-CoNi@Au NRs electrode was introduced in the acidic solution and successive cyclic voltammetry was performed. When the CoNi NRs were not protected with the gold layer, after 5 minutes of immersion in the $\mathrm{H}_{2} \mathrm{SO}_{4}$ solution, the NRs disappear, etched by the acid, and the voltammetry shows the response of the GC (Figure 1c, green line). When the gold layer is not complete, the NRs are also dissolved. Only when the gold layer completely covers the NRs, they (now CoNi@ $\mathrm{Au}$ ) are really protected, even in $\mathrm{H}_{2} \mathrm{SO}_{4}$; in this case, the typical profile of gold in sulphuric solution is obtained (Figure1 c, blue and black lines), even during a high number of scans (more than 20). Therefore, the voltammetric technique allows determining that an immersion time of 8 minutes assures a complete gold layer on the surface of the nanorods. The stability of the CoNi@Au NRs in the aggressive medium supposes the stability of the rods in a less aggressive (cellular) medium. Moreover, the presence of the gold layer is detected by EDS. Figure 1 also shows the composition of the nanorods, before and after the gold recovering. The Au percentage value corresponds to an estimated gold layer of $1.6-2.1 \mathrm{~nm}$. 
(a)

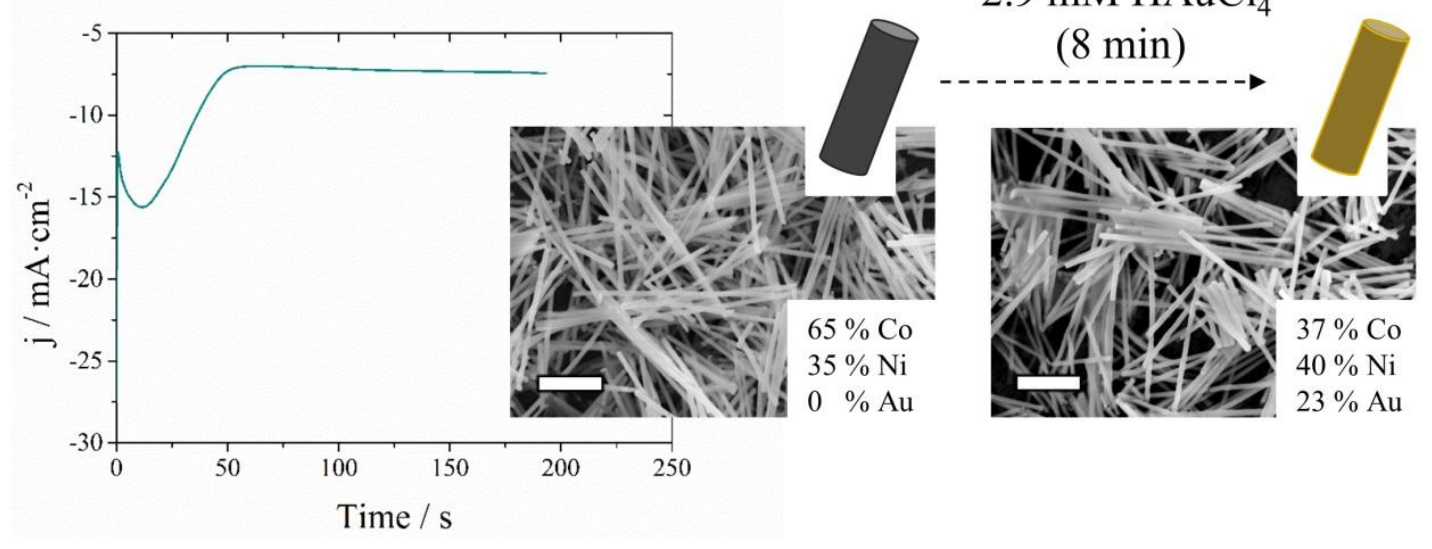

(b)

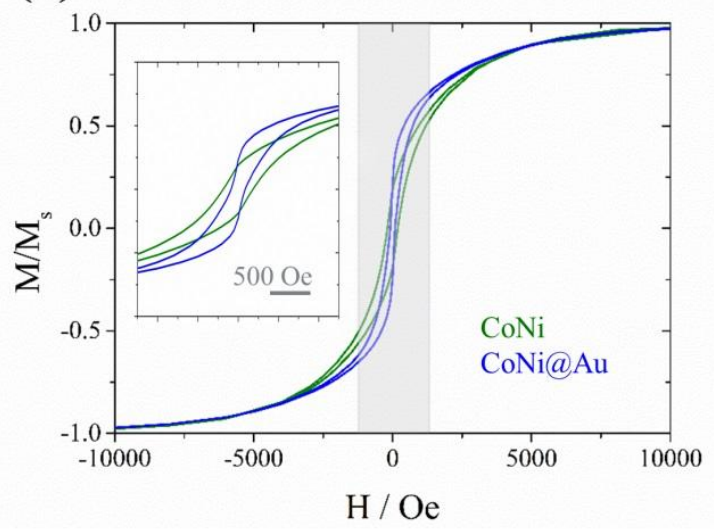

(c)

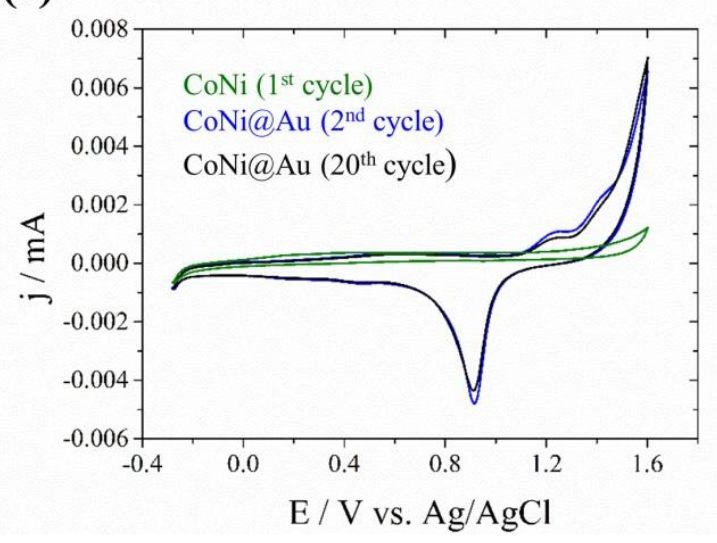

Figure 1. (a) Representative chronoamperometric curve and FE-SEM pictures of CoNi and CoNi@ $\mathrm{Au}$ NRs; Scale bar: $1 \mu \mathrm{m}$. (b) magnetic behaviour of the two types of NRs and (c) cyclic voltammetry at 100 $\mathrm{mV} \mathrm{s}^{-1}$ of the nanorods in $\mathrm{H}_{2} \mathrm{SO}_{4} 0.5 \mathrm{M}$ solution.

The magnetic behaviour of the NRs (CoNi and $\mathrm{CoNi} @ \mathrm{Au})$ was analysed and compared. In both cases, they show a ferromagnetic behaviour, tending to soft-magnetic, with low differences in the curve shape due to the presence of the gold diamagnetic layer (Figure 1b). The value of the magnetization of saturation of the CoNi NRs was $77 \mathrm{emu}^{-1}$, whereas slightly only decreases to $68 \mathrm{emu}^{-1}$ for the CoNi@Au NRs, due to the contribution of the gold layer to the total mass. The coercivity values vary from 150 Oe for the CoNi NRs to 90 Oe for the CoNi@Au NRs and, therefore, CoNi@Au NRs are highly magnetics and easily manipulated by the action of a magnetic field, even with the layer of gold, which makes them promising nanostructures for magnetic drug delivery or magnetic therapies.

The CoNi@Au NRs were easily functionalized through the formation of a self-assembled monolayer by the interaction of thiolate groups, present in MHEG, with the gold surface, as reviewed elsewhere [13]. In this study we chose MHEG because ethylene glycol units improve the solubility of the nanostructures in water as well as their biocompatibility. The presence of MHEG molecules on the CoNi@Au NRs has been corroborated by means of electrochemical probe experiments, by recording cyclic voltammetries of the Fe(II)/Fe(III) in both CoNi@Au and CoNi@Au-MHEG NRs (Figure 2a).

The toxicity of the CoNi@Au-MHEG nanorods was assessed by using HeLa cells, and compared with that of the CoNi-MHEG. Cell viability was evaluated after their incubation with different nanorod concentrations for $24 \mathrm{~h}$ and a posterior post-incubation for another $24 \mathrm{~h}$ with fresh culture medium. Results (Figure 2b) show that the cytotoxic effect is concentration dependent: the cell viability is high, for the two types of NRs (greater than $80 \%$ ) until $10 \mu \mathrm{g} \mathrm{mL}^{-1}$ of nanorods, but when the quantity of nanorods is increased to $52 \mu \mathrm{g} \mathrm{mL}{ }^{-1}$, the cell viability decreases. These results suggest that the CoNi@Au-MHEG 
magnetic nanorods have suitable biocompatibility to be used in biomedical applications. Co-based materials are not usually selected for implants in biomedical applications, due to their potential toxicity; however, their protection with gold and organic molecules makes them potentially useful as drug delivery robots for therapies as cancer treatment. Moreover, the amount of metal delivered to an organism when it is used as drug carrier is very low and the carrier is usually eliminated from the body in a relatively short time. Therefore, CoNi@Au nanostructures could be proposed as promising magnetic nano-carriers for cancer treatments.
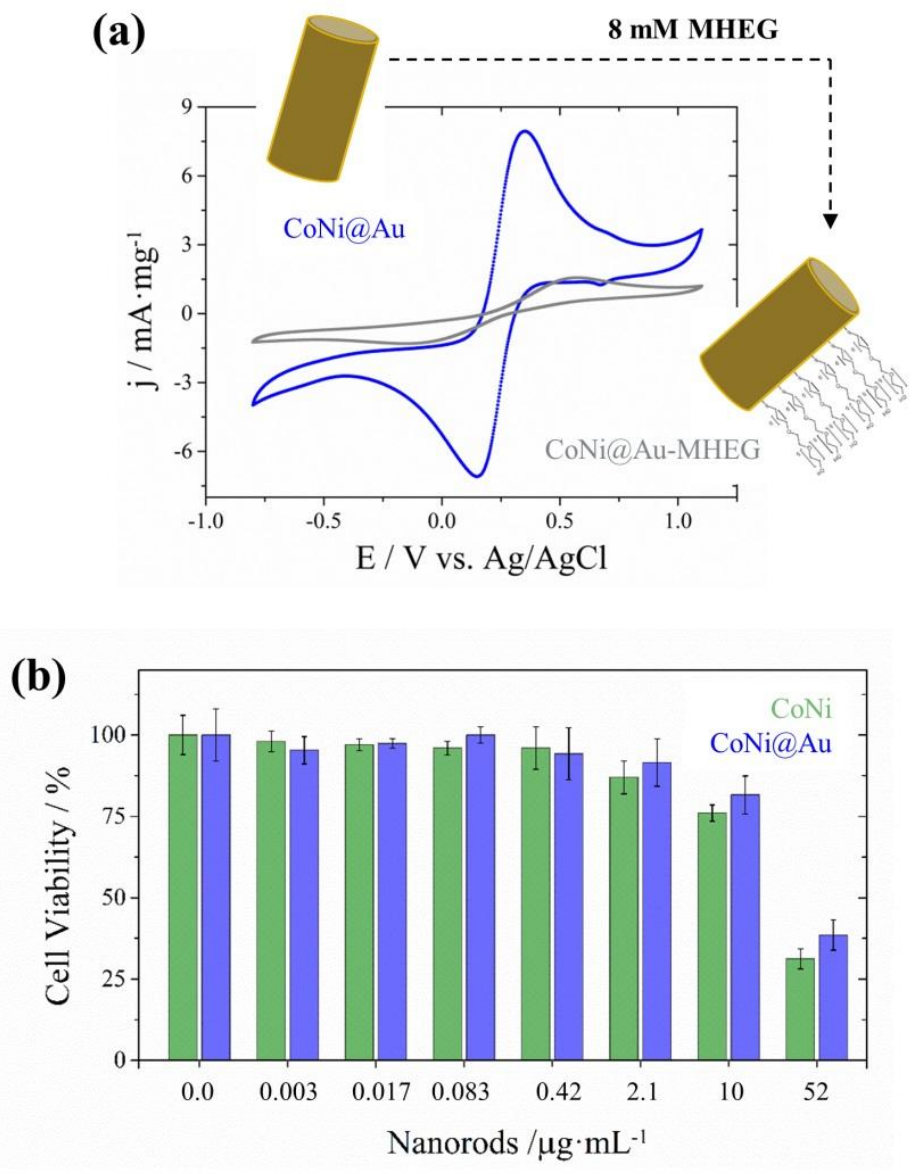

Figure 2. (a) CoNi@Au NRs functionalized with (11-Mercaptoundecyl)hexa(ethylene glycol) and functionalization test by electrochemical probe at $100 \mathrm{mV} \mathrm{s}^{-1}$. (b) HeLa cells viability after incubation with functionalized CoNi-MHEG and CoNi@Au-MHEG NRs at various amounts for $24 \mathrm{~h}$, followed by $24 \mathrm{~h}$ additional incubation with fresh culture medium.

\section{Conclusions}

CoNi@Au magnetic nanorods with good biocompatibility and easy functionalization were synthesised. The CoNi NRs are highly magnetic, with soft-magnetic behaviour and easy magnetic manipulation (fast response to the magnetic field applied). The simple immersion of the nanorods in an $\mathrm{Au}$ (III) solution during a controlled time leads to the formation of a very thin layer of gold on their surface (few nanometers), which confers to the magnetic structures: 1) chemical stability, making them resistant to aggressive media, 2) maintenance of the magnetic manipulation of the nanorods, 3) biocompatibility and 4) ease to functionalize. The CoNi@Au nanorods have been functionalized with (11- 
Mercaptoundecyl)hexa(ethylene glycol), thus forming a hydrophilic layer which helped decrease the magnetic aggregation of the nanowires, and that will be useful for conferring them long circulating properties. A good cellular viability of HeLa cells (>80\%) was obtained up to $10 \mu \mathrm{g}$ nanorods $\mathrm{mL}^{-1}$, which suggests that the prepared nanorods can be further used for anchoring drugs, and for drug targeting and delivery, making them very promising magnetic vehicles for therapy.

\section{ACKNOWLEDGMENT}

This work was supported by the EU ERDF (FEDER) funds and the Spanish Government Grants TEC2011-29140-C03-02 and TEC2014-51940-C2-2-R from MINECO. MEA thanks the Universitat de Barcelona for a predoctoral grant (APIF). Authors thank the CCiTUB for their equipment.

\section{REFERENCES}

[1] M. Hakamada, A, Moriguchi, M. Mabuchi, Fabrication of carbon nanotube/ $\mathrm{NiO}_{x}(\mathrm{OH})_{\mathrm{y}}$ nanocompsite by pulsed electrodeposition for supercapacitor applications, J. Power. Sources, 245 (2014) 324-330.

[2] A. Serrà, E. Gómez, J. F. López-Barbera, J. Nogués, E. Vallés. Green electrochemical template synthesis of CoPt nanoparticles with tunable size, composition, and magnetism from microemulsions using an ionic liquid (bmimPF 6 ), ACS Nano, 8 (2014) 46304639 .

[3] A. Serrà, E. Gómez, E. Vallés, Novel electrodeposition media to synthesize CoNi-Pt core@ shell stable mesoporous nanorods with very high active surface for methanol electro-oxidation, Electrochim. Acta 174 (2015) 630-539.

[4] J. Vilana, M. Lorenzo, E. Gómez, E. Vallés, Electrochemical deposition of CoNi micro/nanostructures as a new materials for electrochemical sensing of glucose, Mater Lett, 159 (2015) 154-158.

[5] M. N. Kozicki, M. Mitkova, M. Park, M. Balakrishnan, C. Gopalan, Information storage using nanoscale electrodeposition of metal in solid electrolytes, Superlattice. Microst. 34 (2003) 459-465.

[6] C.S.S.R. Kumar, F. Mohammad, Magnetic nanomaterials for hyperthermia-based therapy and controlled drug delivery, Adv. Drug. Deliver. Rev., 63 (2011) 789-808.

[7] E. Zhang, M.F. Kircher, M. Koch, L. Eliasson, S.N. Goldberg, E. Renström, Dynamic magnetic fields remote-control apoptosis via nanoparticle rotation, ACS Nano, 8 (2014) 3192-3201.

[8] I. Hilger, W.A. Kaiser, Iron oxide-based nanostructures for MRI and magnetic hyperthermia, Nanomedicine-UK 7 (2012), 1443-1459

[9] J. Chomoucka, J. Drbohlavova, D. Huska, V. Adam, R. Kizek, J. Hubalek, Magnetic nanoparticles and targeted drug delivering, Pharmacol. Res. 62 (2010) 144-149.

[10] K. M. Pondman, A. W. Maijenburg, F. B.Celikkol, A.A. Pathan, U. Kishore, B. Haken, J. E. Elshof, Au coated Ni nanowires with tunable dimensions for biomedical applications, J. Mater. Chem. B, 1 (2013) 6129-6136.

[11] A. Sharma, G. M. Orlowski, Y. Zhu, D. Shore, S. Y. Kim, M. D. DiVito, A. Hubel, B.J H Stadler, Inducing cells to disperse nickel nanowires via integrin-mediated responses, Nanotech. 26 (2015) 135102-135114.

[12] A. O. Fung, V. Kapadia, E. Pierstorff, D. Ho, Y. Chen, Induction of cell death by magnetic actuation of nickel nanowires internalized by fibroblasts, J. Phys. Chem. C, 112 (2008) 15085 -15089.

[13] J. C. Love, L.A. Estroff, J.K. Kriebel, R.G. Nuzzo, G. M. Whitesides, Self-assembled monolayers of thiolates on metal as a form of nanotechnology, Chem. Rev., 105 (2005) 1103-1169. 
1 black and white

Click here to download high resolution image

(a)

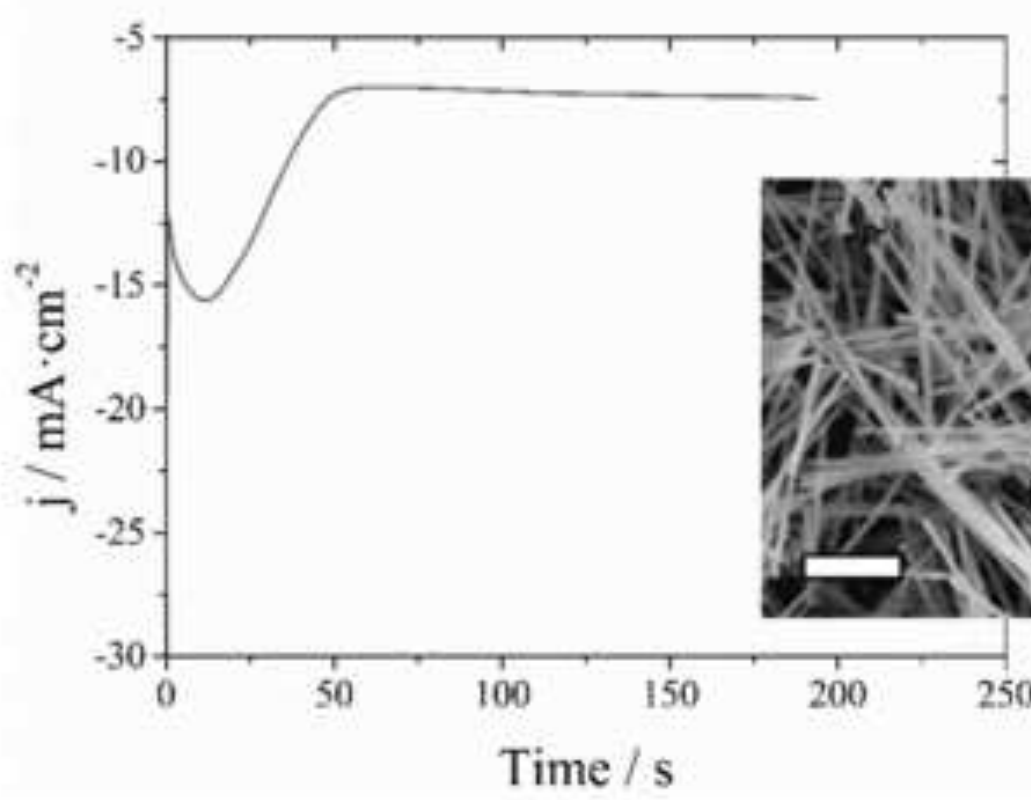

(b)

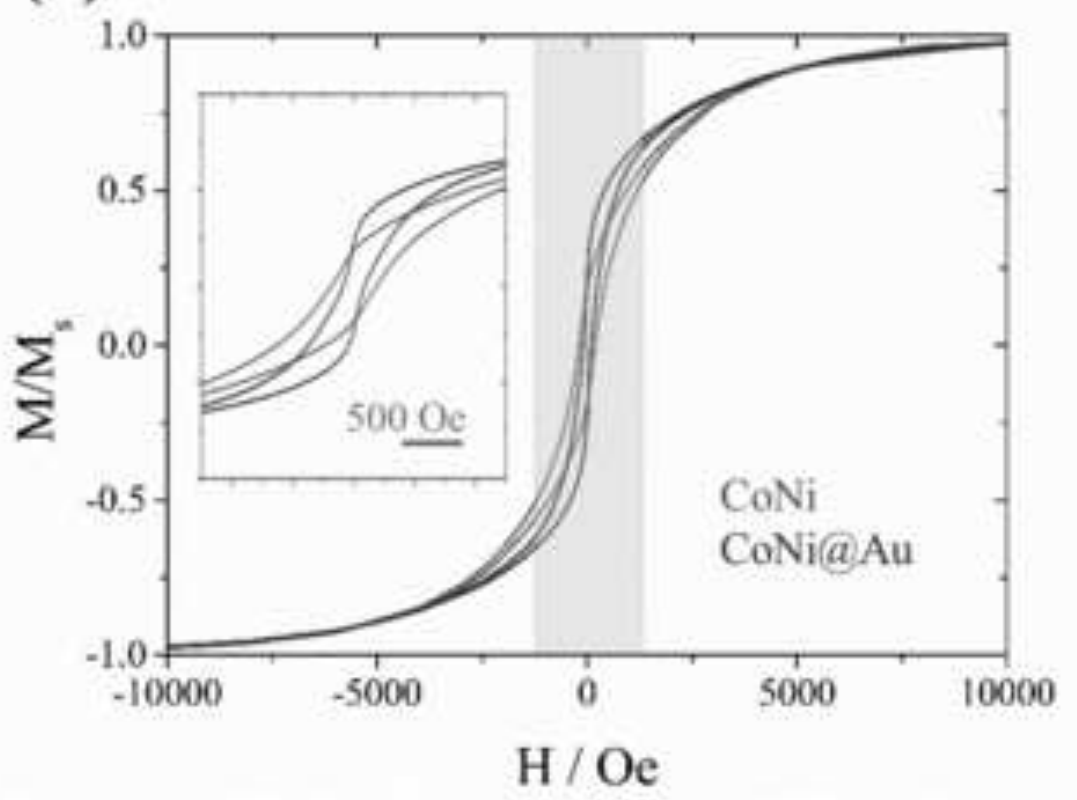

(c)

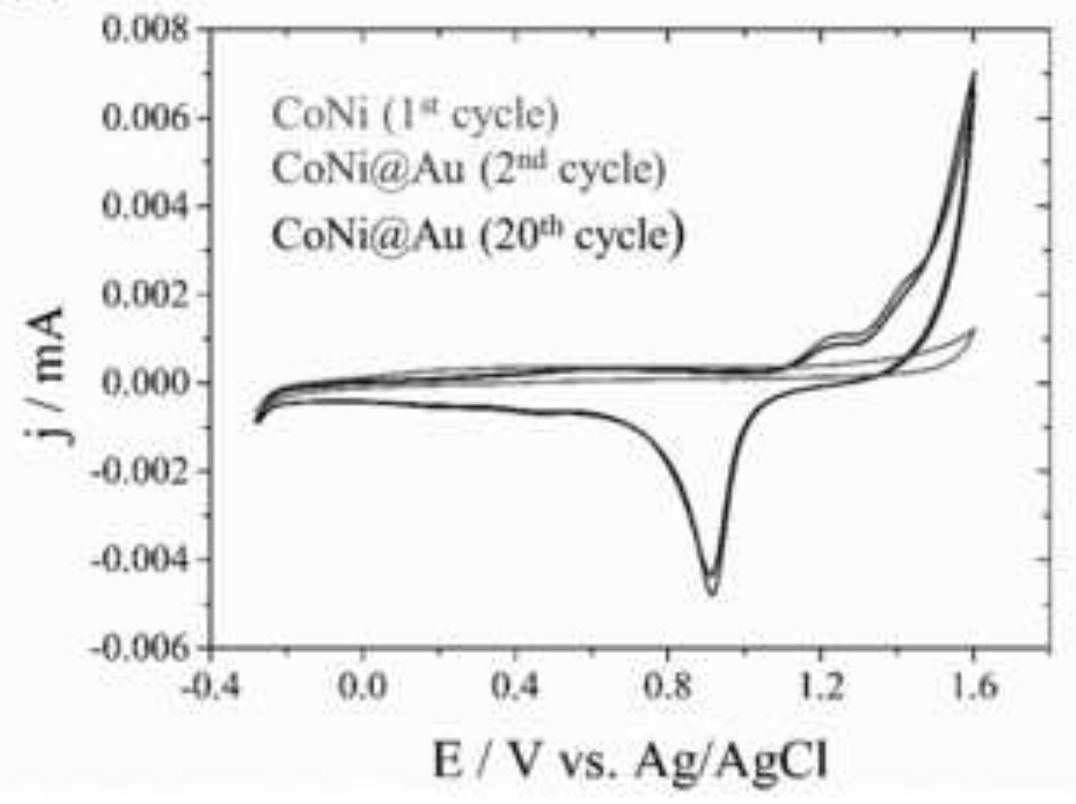



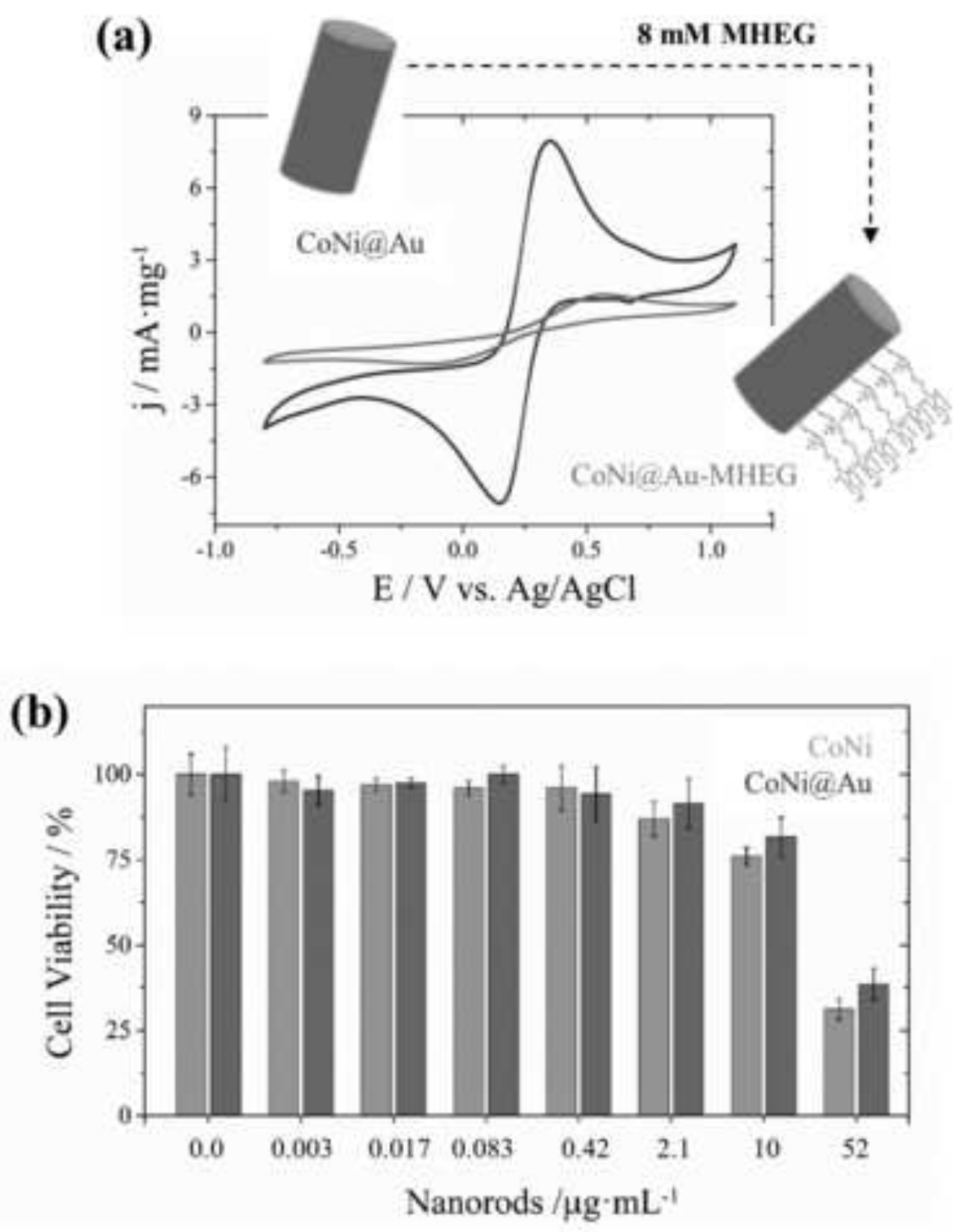